\title{
Correction: BiOsimilaRs in the management of anaemia secondary to chemotherapy in HaEmatology and Oncology: results of the ORHEO observational study
}

Mauricette Michallet ${ }^{1 *}$, Elisabeth Luporsi ${ }^{2}$, Pierre Soubeyran ${ }^{3}$, Nadia Ali Amar $^{4}$, Vincent Boulanger ${ }^{5}$, Miguel Carreiro ${ }^{6}$, Louis-Marie Dourthe ${ }^{7}$, Jean-Luc Labourey ${ }^{5,8}$, Daniel Lepille ${ }^{9}$, Frédéric Maloisel ${ }^{7}$, Jean-Loup Mouysset ${ }^{10}$, Sophie Nahon ${ }^{11}$, Bérengère Narciso ${ }^{12}$, Pierre Nouyrigat ${ }^{13}$, Raouf Radji ${ }^{14}$, Nacéra Sakek ${ }^{15}$, Hélène Albrand ${ }^{16}$ and The ORHEO study group

\section{Correction}

After publication of this Research Article [1], we noted errors in Table 1, and in the rate of thromboembolic events (page 7). Specifically, in Table 1 the percentage of female and male patients were incorrectly given as $7 \%$ and $3 \%$ respectively. The correct figures are $48.6 \%$ female and $51.4 \%$ male (please see a corrected version of Table 1 below, and the Additional file 1). The correct rate of thromboembolic events is $3.49 \%$ (originally stated as $3.55 \%)$.

\section{Table 1 Patient demographics}

\begin{tabular}{lc}
\hline Characteristic & Value \\
\hline Age, mean \pm SD (years) & $66.49 \pm 11.77$ \\
Patients with solid tumours, mean (years) & 65.76 \\
Patients with lymphoma, mean (years) & 68.59 \\
Patients with myeloma, mean (years) & 70.65 \\
Female $(\%)$ & 48.6 \\
Male $(\%)$ & 51.4 \\
Hb, mean $\pm S D(g / d L)$ & $9.59 \pm 0.88$ \\
sBP, mean $\pm S D(m m H g)$ & $125.75 \pm 14.48$ \\
dBP, mean $\pm S D(m m H g)$ & $74.18 \pm 9.79$ \\
Malignancies, $\mathrm{n}(\%)$ & $2310(100)$ \\
Solid tumours, $\mathrm{n}(\%)$ & $1838(79.57)$ \\
Lymphoma, $\mathrm{n}(\%)$ & $301(13.03)$ \\
Myeloma, $\mathrm{n}(\%)$ & $171(7.40)$ \\
Height, mean $\pm \mathrm{SD}(\mathrm{cm})$ & $166.35 \pm 8.72$ \\
Weight, mean $\pm \mathrm{SD}(\mathrm{kg})$ & $67.46 \pm 14.23$ \\
BMl, mean $\pm \mathrm{SD}\left(\mathrm{kg} / \mathrm{m}^{2}\right)$ & $24.33 \pm 4.62$
\end{tabular}

$B M I$ body mass index, $A B P$ diastolic blood pressure, $H b$ haemoglobin, $s B P$ systolic blood pressure, $S D$ standard deviation.

\footnotetext{
* Correspondence: Mauricette.michallet@chu-lyon.fr

'Centre Hospitalier Lyon Sud, Lyon, France

Full list of author information is available at the end of the article
} 


\section{Additional file}

Additional file 1: Corrected version of article BMC Cancer 14:503.

\section{Author details}

${ }^{1}$ Centre Hospitalier Lyon Sud, Lyon, France. ${ }^{2}$ Centre Alexis Vautrin, Vandoeuvre-les-Nancy, France. ${ }^{3}$ Institut Bergonie, Bordeaux, France. ${ }^{4}$ Centre Hospitalier de Troyes, Troyes, France. ${ }^{5}$ Centre Hospitalier de Carcassonne, Carcassonne, France. ${ }^{6}$ Centre Hospitalier de Montauban, Montauban, France. ${ }^{7}$ Clinique Sainte Anne, Strasbourg, France. ${ }^{8}$ Polyclinique Montréal, Carcassonne, France. ${ }^{9}$ Clinique Pasteur, Evreux, France. ${ }^{10}$ Clinique Rambot-Provencale, Aix en Provence, France. ${ }^{11}$ Centre Hospitalier d'Aix en Provence, Aix en Provence, France. ${ }^{12}$ Centre Hospitalier Régional Universitaire de Tours, Tours, France. ${ }^{13}$ Clinique du Cap d'Or, La Seyne sur Mer, France.

${ }^{14}$ Centre Frédéric JOLLIOT, Rouen, France. ${ }^{15}$ Centre Hospitalier de Montbéliard, Montbeliard, France. ${ }^{16}$ Laboratoire HOSPIRA France, MeudonLa Foret, France.

Received: 3 September 2014 Accepted: 18 September 2014 Published: 26 September 2014

\section{Reference}

1. Michallet M, Luporsi E, Soubeyran P, Amar NA, Boulanger V, Carreiro M, Dourthe L-M, Labourey J-L, Lepille D, Maloisel F, Mouysset J-L, Nahon S, Narciso B, Nouyrigat P, Radji R, Sakek N, Albrand H: BiOsimilaRs in the management of anaemia secondary to chemotherapy in HaEmatology and Oncology: results of the ORHEO observational study. BMC Cancer 2014, 14:503.

doi:10.1186/1471-2407-14-720

Cite this article as: Michallet et al:: Correction: BiOsimilaRs in the management of anaemia secondary to chemotherapy in HaEmatology and Oncology: results of the ORHEO observational study. BMC Cancer 2014 14:720.

\section{Submit your next manuscript to BioMed Central and take full advantage of:}

- Convenient online submission

- Thorough peer review

- No space constraints or color figure charges

- Immediate publication on acceptance

- Inclusion in PubMed, CAS, Scopus and Google Scholar

- Research which is freely available for redistribution 